Poster presented at 8th International

Conference on Instrumentation for Colliding Beam Physics, Novosibirsk, Russia, Feb-Mar 2002.

\title{
Cesium Monitoring System for ATLAS Tile Hadron Calorimeter
}

\author{
E. Starchenko ${ }^{a, *}$, G. Blanchot ${ }^{\mathrm{d}}$, M. Bosman ${ }^{\mathrm{d}}$, \\ M. Cavalli-Sforza ${ }^{\text {d}}$, A. Karyukhin ${ }^{a}$, S. Kopikov ${ }^{a}$, A. Miagkov ${ }^{a}$, \\ M. Nessi ${ }^{c}$, A. Shalimov ${ }^{a}$, N. Shalanda ${ }^{a}$, M. Soldatov ${ }^{\text {a }}$, \\ A. Solodkov ${ }^{a}$, A. Soloviev ${ }^{\text {b }, ~ V . ~ T s o u p k o-S i t n i k o v ~}{ }^{b}$, A. Zaitsev ${ }^{\text {a }}$ \\ a IHEP, Protvino, Russia \\ b JINR, Dubna, Russia \\ ${ }^{\mathrm{c}}$ CERN, Geneva, Switzerland \\ dIFAE, Barcelona, Spain
}

For the ATLAS / Tile Calorimeter Collaboration

\begin{abstract}
A system to calibrate and monitor ATLAS Barrel Hadronic Calorimeter (TileCal)) is under construction at CERN Laboratory. A movable radioactive source driven by a liquid flow travels through the calorimeter body deposing a known energy to the calorimeter cells. Extensive R\&D studies have been carried out and the main system parameters are evaluated. The prototypes are currently used for quality check and inter-calibration of the TileCal modules. A distributed control system, hardware as well as corresponding on-line and off-line software is developed.
\end{abstract}

\section{Introduction}

ATLAS is a general-purpose $p p$ experiment at the Large Hadron Collider (LHC) to be built at CERN [1]. The Tile Hadron Calorimeter (TileCal) is an essential part of the detector, contributing to energy and missing $\mathrm{p}_{T}$ measurements in $14 \mathrm{TeV} p p$ interactions.

* Corresponding author. E-mail address:starchenko@mx.ihep.su 
TileCal is a sampling calorimeter made of steel plates (absorber) and scintillating tiles (active material). The design, general features and expected performance of the calorimeter are well described in the "ATLAS Tile Calorimeter Technical Design Report" [2]. It consists of one barrel and two extended barrel parts. All the three sections have a cylindrical structure further subdivided into 64 independent modules. The cells are defined by grouping together sets of optical fibers into bunches leading to PMTs.

A system using a movable radioactive source was proposed for TileCal calibration and monitoring. In the system (MonSys) a capsule containing $\mathrm{Cs}^{137}$ $\gamma$-source are carried along by a liquid flow inside calibration tubes piercing through all the cells of the calorimeter [2] (Fig. 1). The corresponding variation of a PMT current with the capsule path reflects the tiles and fibers optical quality in the cell. The read-out principle for the PMT current measurements is described elsewhere [3].

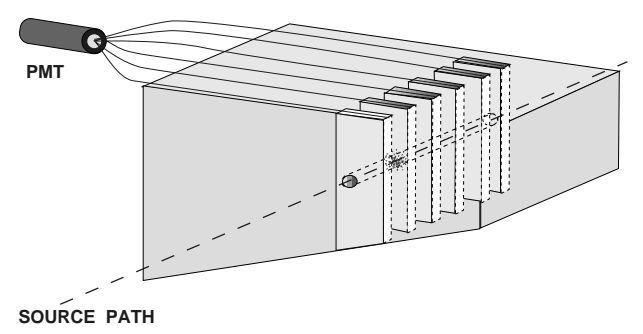

Fig. 1. Cs MonSys concept

\section{MonSys Parameters}

A number of R\&D studies were carried out at IHEP and MonSys main design parameters evaluated. Further tests at CERN confirmed the correctness of the approach.

Calibration tubes: with inner diameters from 4.0 to $6.8 \mathrm{~mm}$ were tried and a stainless steel tube of $\oslash 6.0 \times 8.0 \mathrm{~mm}$ was accepted; the total length of all the tubes, including the straight and bent parts, is close to $10 \mathrm{~km}$ for the all three TileCal subsystems.

Working media: during most of the tests plain water was used. Some studies were done using "fluorocarbon" liquid (e.g. $\mathrm{C}_{6} \mathrm{~F}_{14}$ ) as propagated liquid. It was proved that the system can use this type of working media. Water was accepted for the further use in the system as the best and cheapest solution. Up to $400 \mathrm{l}$ of water will be used for the whole MonSys.

Source capsule: hardened aluminium alloy protected with 2-4 microns of TiN 
film was chosen for the source capsule body (Fig. 2). The dumb-bell shape and the outside dimensions of the capsule $(\oslash 5.6$ by $11.5 \mathrm{~mm})$ allow it to be driven through bent tube sections with curvature radius down to $15 \mathrm{~mm}$ ). The source itself is enclosed in tightly welded stainless steel tube [5].

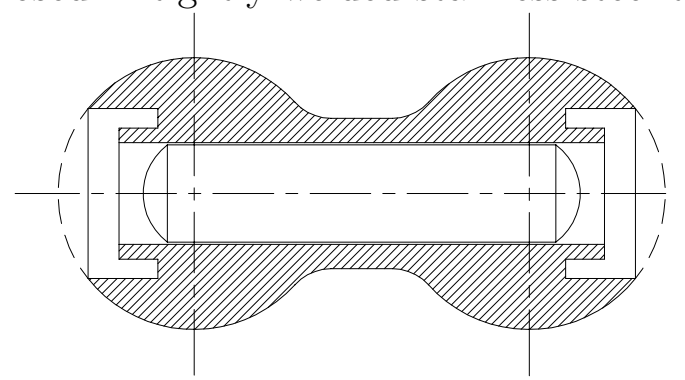

Fig. 2. The design of the source capsule

Capsule cruising speed: velocities from 5 to $60 \mathrm{~cm} / \mathrm{s}$ were tried and a working speed range of about $25-30 \mathrm{~cm} / \mathrm{s}$ was chosen. The calibration time is less than 5 hours for the barrel part of the calorimeter and 3 hours for the extended ones. All three TileCal sections will be calibrated in parallel using separate sources.

Capsule location sensors: two basic principles of capsule location sensors were considered: optical detection and electromagnetic one. SIN type (Sensor based on INductance) was chosen for further use [4]. That is a coil located on a tube which registers a metal capsule moving inside by the base oscillating frequency shift. Frequency variation is detected with PLL circuit and used as a trigger (Fig. 3).

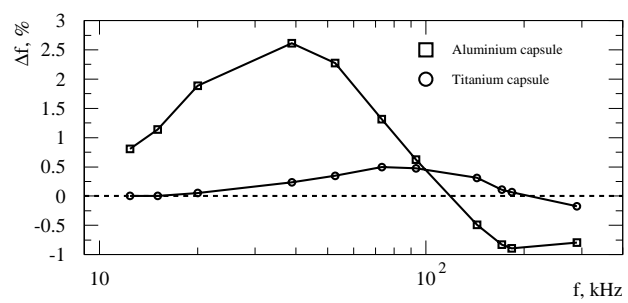

Fig. 3. Frequency variation caused by a capsule

Source storage device ("Garage"): a mechanical device where the source normally resides between the calibration runs. Means of control (SIN type sensors and Geiger counter) were tested in real run conditions, design and principles were fixed. Seven such devices supposed to be used in total in TileCal.

\section{Design and Prototyping}

A diagram of MonSys prototype hydraulics is given in Fig. 4. The main feature of the proposed scheme is that the liquid moves, driving the source, only in 
a small part of the system (in the contour of two or four modules); thus the applied dynamic pressure during the run remains at a rather low value.

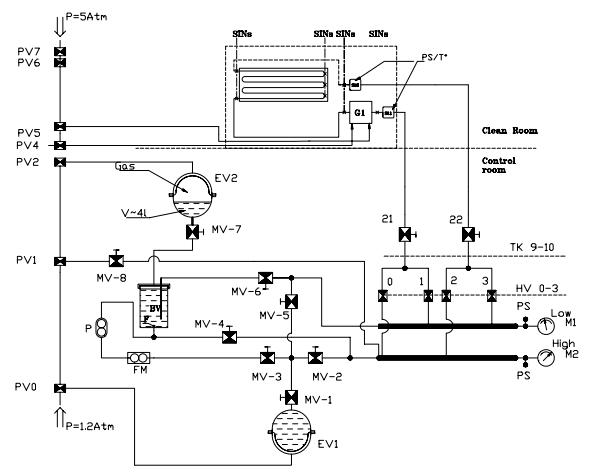

Fig. 4. A diagram of a MonSys prototype hydraulic layout

At present three prototypes of the MonSys hydraulic equipment setups are built at CERN. They are used to provide a daily quality check of the modules being instrumented as well as to calibrate, inter-calibrate and monitor the TileCal modules during beam tests. Fig. 5 shows a barrel module under check at CERN. Bent tubes with SIN type sensors on them, and the flexible pipe connecting the hydraulic drive to the calibration tube joint, can be seen too.

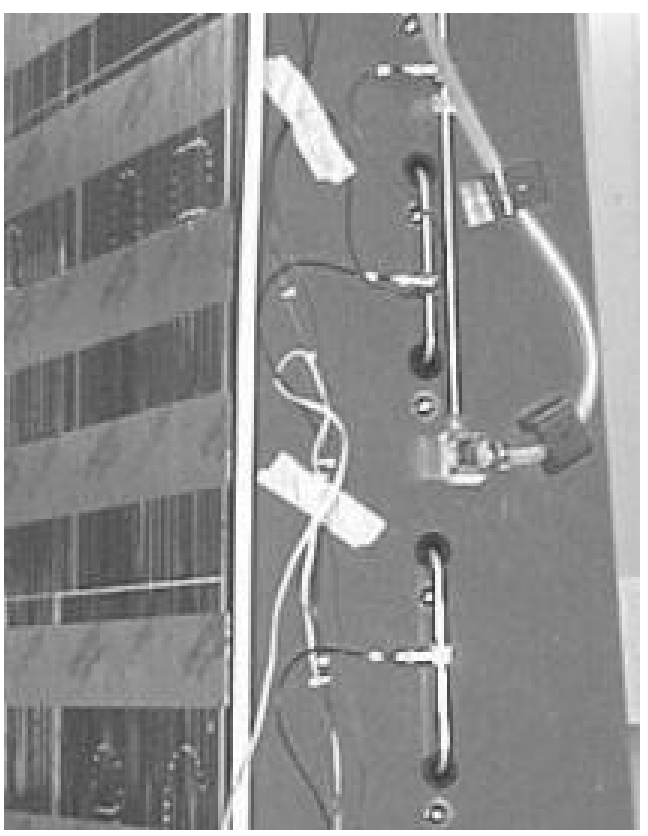

Fig. 5. A TileCal barrel module under check during the instrumentation

It takes about 5 minutes to run the source through a module. The quality of the collected data is checked on-line and the data treatment starts. In Fig. 6 an example of calibration data off-line treatment is given for a case when one of a fiber in a cell is broken.

The accuracy of a single tile response evaluation is better than $2 \%$. That 


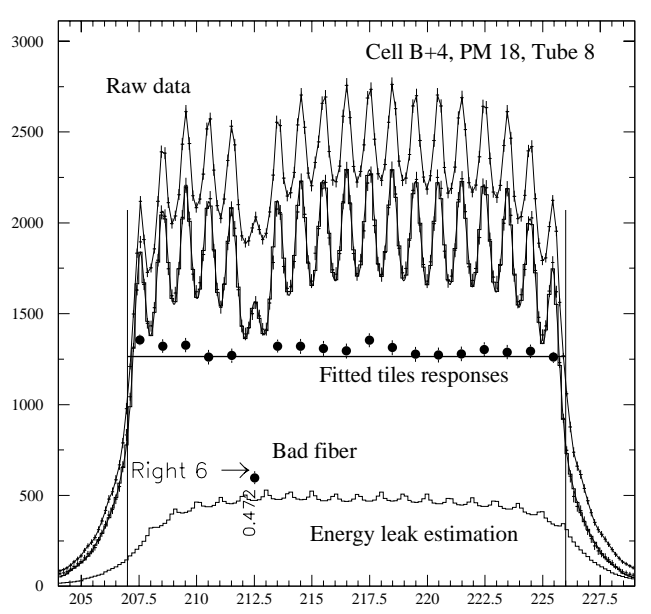

Fig. 6. Calibration data treatment. The accuracy of a single tile response value is close to $1.8 \%$

leads to a tile row mean response accuracy close to $0.6 \%$ and $0.3 \%$ for a cell in average. The accuracy is enough to calibrate and monitor TileCal.

\section{Acknowledgements}

The authors are very grateful to all the Collaboration members who took a part in all the discussions, talks, tests and in the intensive use of the Cs monitoring system during the last years.

\section{References}

[1] ATLAS Technical Proposal, CERN/LHCC/94-43 LHCC/P2, 15-Dec-1994;

[2] TileCal Technical Design Report, CERN/LHCC 96-42, 15-Dec-1996;

[3] G.Blanchot et al., ATLAS Internal Note, TILECAL-94-044, 20-Dec-1994; G.Blanchot et al., ATLAS Internal Note, TILECAL-94-045, 20-Dec-1994;

[4] A.Karyukhin et al., ATLAS Internal Note, TILECAL-98-134, 17-Nov-1997;

[5] J.Budagov et al., Radioactive sources for ATLAS hadron tile calorimeter calibration, JINR preprint E13-97-104 (1997). 